\title{
McKAY QUIVERS AND EXTENDED DYNKIN DIAGRAMS
}

\author{
BY \\ MAURICE AUSLANDER ${ }^{1}$ AND IDUN REITEN ${ }^{2}$
}

\begin{abstract}
Let $k$ be an algebraically closed field and $G$ a finite nontrivial group whose order is not divisible by the characteristic of $k$. Associated with an $m$-dimensional representation of $G$ is the McKay quiver, whose vertices correspond to the irreducible representations of $G$. We show that if $m=2$, then the underlying graph of the separated McKay quiver is a finite union of extended Dynkin diagrams.
\end{abstract}

Introduction. Throughout this paper let $k$ denote an algebraically closed field and $G$ a finite nontrivial group whose order is not divisible by the characteristic of $k$. Denote by $V_{1}, \ldots, V_{n}$ the nonisomorphic irreducible representations of $G$ over $k$. Associated with a fixed $m$-dimensional representation $V$ of $G$ (or with a group homomorphism $G \rightarrow \mathrm{GL}(m, k))$, there is the following quiver, that is, a set of vertices with arrows between the vertices. The vertices are in one-one correspondence with the $V_{i}$, and if we write $V \otimes_{k} V_{i}=\coprod_{j=1}^{n} t_{i j} V_{j}$, there are $t_{i j}$ arrows from $V_{j}$ to $V_{i}$. This quiver is called the McKay quiver associated with $(G, V)$, or with $G \rightarrow \mathrm{GL}(m, k)$.

An interesting occurrence of the extended Dynkin diagrams for these McKay quivers was observed by McKay [10]: If in the McKay quiver for $G \subset \mathrm{SL}(2, \mathbf{C})$, where $\mathbf{C}$ denotes the complex numbers, each $\cdot \rightleftarrows$. is replaced by $\cdot-\cdot$, we have one of the extended Dynkin diagrams $\tilde{A}_{n}, \tilde{D}_{n}, \tilde{E}_{6}, \tilde{E}_{7}, \tilde{E}_{8}$, which occur respectively for the cyclic, binary dihedral, binary tetrahedral, binary octahedral and binary icosahedral groups. Furthermore, the corresponding Dynkin diagram is the same as the one occurring in the minimal resolution of singularities for the quotient surface $\mathbf{C}^{2} / G$ (see $\left.[\mathbf{3}]\right)$. Various explanations have been given for this occurrence of extended Dynkin diagrams, some of which at the same time explain the connection with resolution of singularities $[\mathbf{7}, \mathbf{1 2}, \mathbf{6}, \mathbf{8}]$.

The aim of this note is to prove a generalization of McKay's observation to arbitrary two-dimensional representations. We recall that if $Q$ is a quiver with vertices $v_{1}, \ldots, v_{n}$, then the separated quiver $\bar{Q}$ has as vertices $v_{1}, \ldots, v_{n}, v_{1}^{\prime}, \ldots, v_{n}^{\prime}$, and for each arrow $\alpha: v_{i} \rightarrow v_{j}$ in $Q$, we have an arrow $\bar{\alpha}: v_{i} \rightarrow v_{j}^{\prime}$ in $\bar{Q}$. We prove that the underlying graph $|\bar{Q}|$ of the separated McKay quiver $Q$ for $G \rightarrow \mathrm{GL}(2, k)$ is a disjoint union of extended Dynkin diagrams. This has been proved independently by Lenzing (private communication). Also for $G \rightarrow \mathrm{GL}(m, k)$ with $m>2$, this is never the case. Based upon simple combinatorics and some properties of subgroups of SL $(2, k)$, we explain why McKay's observation can be regarded as a special case

Received by the editors February 21, 1985. This paper was presented by the second author at the Southern California Ring Theory meeting at U.S.C. in December 1984.

1980 Mathematics Subject Classification. Primary 16A64; Secondary 20C15.

${ }^{1}$ Partially supported by NSF Grant MCS 8303348 .

${ }^{2}$ Partially supported by the Norwegian Research Council and NSF (MCS 8303348). 
of our result, and we also study the relationship with the work of Happel, Preisel and Ringel [7]. It is interesting that this occurrence of extended Dynkin diagrams is intimately connected with their occurrence for tame hereditary finite dimensional $k$-algebras.

In the theory of finite dimensional $k$-algebras the study of quivers plays an important role. With a $k$-algebra $\Lambda$ there is associated a (Gabriel) quiver in the following way. The vertices correspond to the nonisomorphic indecomposable projective $\Lambda$ modules. The number of arrows from the vertex of $P$ to the vertex of $Q$ is the multiplicity of $Q / \mathbf{r} Q$ in $\mathbf{r} P / \mathbf{r}^{2} P$, where $\mathbf{r}$ denotes the radical of $\Lambda$, or equivalently, the multiplicity of $Q$ in $E$, where $E \rightarrow P \rightarrow P / \mathbf{r} P \rightarrow 0$ is a minimal projective presentation. In particular there is a one-one correspondence between the quivers with a finite number of vertices and arrows and the basic $k$-algebras with $\mathbf{r}^{2}=0$. Here the $k$-algebra associated with a quiver is the path algebra modulo the relations generated by composition of two paths. This way there is a radical square zero algebra associated with $(G, V)$, via the McKay quiver. In addition to using work of Auslander [1] on the connection between McKay quivers and skew group rings, it is this interpretation which enables us to use results on finite dimensional algebras to prove our result.

There is also another type of quiver, the AR-quiver, often associated with a module category over a ring, in the cases where almost split sequences exist for certain subcategories, like for the category of finitely generated modules in the case of finite dimensional $k$-algebras. For these quivers there are heavy restrictions on which ones can occur, and answering such questions is interesting for classification problems. It is interesting that McKay quivers also have this other connection with ring theory, as they in some cases can be interpreted as AR-quivers. Hence the computation of McKay quivers is interesting from this point of view. For Auslander has shown that the AR-quivers for the reflexive modules over the fixed ring $k[[X, Y]]^{G}$, with the natural action of $G$, is isomorphic to the McKay quiver when $G \subset \mathrm{GL}(2, k)$ is a finite subgroup with no pseudoreflections [1]. By giving the McKay quiver a natural structure of translation quiver, we get an isomorphism of translation quivers. As an illustration of our results we give the structure of these McKay quivers.

Theorem 1 was announced at ICRA IV in Ottawa 1984. We would like to thank J. Carlson for discussions on some of the material in $\S 2$.

1. The main result. The principal aim of this section is to prove

THEOREM 1. Let $k$ be an algebraically closed field and $G$ a finite nontrivial group whose order is not divisible by the characteristic of $k$. If $Q$ denotes the $M c K a y$ quiver of some $G \rightarrow \mathrm{GL}(m, k)$, we have the following:

(a) If $m=2$, then the underlying graph $|\bar{Q}|$ of the separated McKay quiver $\bar{Q}$ is a finite disjoint union of copies of the extended Dynkin diagrams $\tilde{A}_{n}, \tilde{D}_{n}, \tilde{E}_{6}, \tilde{E}_{7}, \tilde{E}_{8}$.

(b) If $m>2,|\bar{Q}|$ is a disjoint union of graphs, which are neither Dynkin nor extended Dynkin.

To prove this theorem we need the following lemmas, the first one being a direct consequence of $[\mathbf{1}, \S 1]$, and the other two dealing with finite dimensional algebras. We recall that if $G$ is a finite group acting on a ring $\Lambda$, then the skew group ring $\Lambda G$ has the same elements and addition as the ordinary group ring, and multiplication 
is induced by $(\lambda g)\left(\lambda^{\prime} g^{\prime}\right)=\lambda g\left(\lambda^{\prime}\right) g g^{\prime}$. We also point out that $G \rightarrow \mathrm{GL}(m, k)$ gives a natural action of $G$ on $S=k\left[\left[X_{1}, \ldots, X_{m}\right]\right]$, and hence also on $\Lambda=S /(\operatorname{rad} S)^{2}$.

LEMMA 2. Let $\Lambda=k\left[\left[X_{1}, \ldots, X_{m}\right]\right] /\left(X_{1}, \ldots, X_{m}\right)^{2}$ with $G$ acting as induced from $G \rightarrow \mathrm{GL}(m, k)$ and such that the order of $G$ is not divisible by the characteristic of $k$. Then the skew group ring $\Lambda G$ is Morita equivalent to the radical square zero algebra given by the opposite of the McKay quiver for $G \rightarrow \mathrm{GL}(m, k)$.

ProOF. This follows directly from $[\mathbf{1}]$, since $(\operatorname{rad} S) S G=\operatorname{rad} S G$, and hence $S G /(\operatorname{rad} S G)^{2} \simeq S /(\operatorname{rad} S)^{2} G$. This shows that $\Lambda G$ and $S G$ have the same associated quiver, and the quiver for $S G$ is shown in $[\mathbf{1}, \S 1]$ to be the opposite of the McKay quiver for $G \rightarrow \mathrm{GL}(m, k)$.

LEMMA 3. Let $\Lambda$ be a basic finite dimensional $k$-algebra with $\mathbf{r}^{2}=0$, and $G$ a finite group acting on $\Lambda$ whose order is not divisible by the characteristic of $k$. Denoting by $\Gamma_{\Lambda}$ the ring $\left(\begin{array}{cc}\Lambda / \mathbf{r} & 0 \\ \mathbf{r} & \Lambda / \mathbf{r}\end{array}\right)$, and considering the induced action of $G$ on $\Gamma_{\Lambda}$, we have $\Gamma_{\Lambda} G \simeq \Gamma_{\Lambda G}$.

ProOF. We use that since the order of $G$ is invertible in $\Lambda, \operatorname{rad} \Lambda G=\mathbf{r} \Lambda G=\mathbf{r} G$ and $(\Lambda / \mathbf{r}) G \simeq \Lambda G / \operatorname{rad} \Lambda G$.

LEMMA 4. Let $\Lambda$ be an indecomposable finite dimensional path algebra of a quiver $Q$ over $k$ and $G$ a finite group acting on $\Lambda$ such that the order of $G$ is not divisible by the characteristic of $k$.

(a) If $|Q|$ is extended Dynkin, then every indecomposable ring summand of $\Lambda G$ is Morita equivalent to the path algebra of an extended Dynkin diagram.

(b) If $|Q|$ is neither Dynkin nor extended Dynkin, then any indecomposable ring summand of $\Lambda G$ is Morita equivalent to the path algebra of a quiver $Q^{\prime}$, where $\left|Q^{\prime}\right|$ is neither Dynkin nor extended Dynkin.

PROOF. The finite dimensional path algebras over $k$ are known to be exactly the basic finite dimensional hereditary $k$-algebras. We denote by $D$ the ordinary duality and by $\operatorname{Tr}$ the transpose, and recall that for an hereditary algebra $\Lambda$ an indecomposable $\Lambda$-module is said to be preprojective if it is of the form $(\operatorname{Tr} D)^{i} P$ for $P$ projective, and preinjective if it is of the form $(D \operatorname{Tr})^{i} I$ for $I$ injective $[4] . C$ is $D$ Tr-periodic if $(D \operatorname{Tr})^{i} C \simeq C$ for some $i>0$. It is known that $|Q|$ is extended Dynkin if and only if every indecomposable finitely generated $\Lambda$-module is preprojective, preinjective or $D$ Tr-periodic, and for each noninjective indecomposable projective $P$ no $(\operatorname{Tr} D)^{i} P$ is injective (see $[2]$ ). It is further known that a finitely generated $\Lambda$-module $C$ is projective (injective) if and only if $\Lambda G \otimes_{\Lambda} C$ is, and we know from [11] that if $0 \rightarrow A \rightarrow B \rightarrow C \rightarrow 0$ is an almost split sequence, then $0 \rightarrow \Lambda G \otimes_{\Lambda} A \rightarrow \Lambda G \otimes_{\Lambda} B \rightarrow \Lambda G \otimes_{\Lambda} C \rightarrow 0$ is a direct sum of almost split sequences. Further we know that $A \simeq D \operatorname{Tr} C$ when $0 \rightarrow A \rightarrow B \rightarrow C \rightarrow 0$ is almost split, that a $\Lambda$-module $C$ is a direct summand of the $\Lambda$-module $\Lambda G \otimes_{\Lambda} C$, and that every indecomposable $\Lambda G$-module is a summand of some $\Lambda G \otimes_{\Lambda} C$. The proof follows easily by combining these facts.

If $\Lambda$ is a finite dimensional $k$-algebra with $\mathbf{r}^{2}=0$, it is well known and easy to see that the quiver for $\Gamma_{\Lambda}$ is the separated quiver for $\Lambda$ (and that $\Gamma_{\Lambda}$ is an hereditary algebra stably equivalent to $\Lambda)$. The quiver $Q$ for $\Lambda=k\left[X_{1}, \ldots, X_{m}\right] /\left(X_{1}, \ldots, X_{m}\right)^{2}$ is

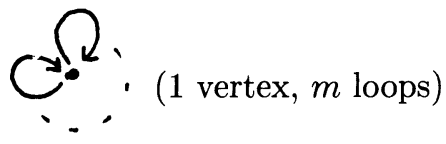


and for $\Gamma_{\Lambda}$ is

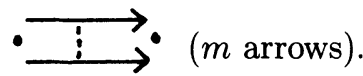

The separated quiver for $\Lambda G$ is the quiver of $\Gamma_{\Lambda G} \simeq\left(\Gamma_{\Lambda}\right) G$. Our claim now follows, since $|Q|$ is extended Dynkin if $m=2$ and neither Dynkin nor extended Dynkin if $m>2$. This finishes the proof of the theorem.

We next show that McKay's observation can be deduced from Theorem 1 by using some group theoretic facts in addition to a combinatorial argument, so that our result can be viewed as a generalization. We also recover the diagrams in [7] for the case of a selfdual representation, in the algebraically closed case. For this the following combinatorial lemma is essential.

LEMMA 5. Let $Q$ be a connected quiver such that for any two vertices $v$ and $w$, the number of arrows from $v$ to $w$ is the same as the number of arrows from $w$ to $v$, and denote by $\|Q\|$ the graph obtained by replacing each pair

$$
\text { • } \cdot \quad \text { by - . } . \quad \text { and } \mathrm{G} \text { by }
$$

(a) If $Q$ has no loops $\bigcap_{\text {and }}|\bar{Q}|$ is a disjoint union of extended Dynkin diagrams, then $\|Q\|$ is an extended Dynkin diagram.

(b) If $|\bar{Q}|$ is a disjoint union of extended Dynkin diagrams, then $\|Q\|$ is an extended Dynkin diagram or of the form

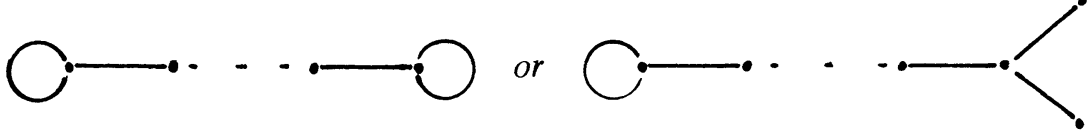

Proof. (a) If $\|Q\|$ is a tree (with single edges), it is easy to see that $|\bar{Q}|$ is a disjoint union of two copies of the same tree. If $\|Q\|$ contains a cycle, which by assumption is not a loop, then clearly $|\bar{Q}|$ contains a cycle, and if one of the vertices in the cycle in $\|Q\|$ is connected with a vertex not in the cycle, the same will be the case for $|\bar{Q}|$. Since $Q$ is assumed to be connected, the claim follows.

(b) Assume that $Q$ has loops, and let $Q^{\prime}$ denote the quiver obtained from $Q$ by removing the loops. It is easy to see that when $|\bar{Q}|$ is a disjoint union of extended Dynkin diagrams $\left\|Q^{\prime}\right\|$ cannot contain any cycle, so that $\left\|Q^{\prime}\right\|$ must be a tree, and $\left|\bar{Q}^{\prime}\right|$ is a union of two copies of this tree, which must be a proper subdiagram of an extended Dynkin diagram. For each loop in $Q$ there will be an edge connecting the two trees in $|\bar{Q}|$. Since $|\bar{Q}|$ is extended Dynkin, it is easy to see that the claim follows.

Now let $Q$ be the McKay quiver of a finite nontrivial subgroup $G$ of $\operatorname{SL}(2, k) . Q$ is connected because $G$ is a subgroup of $\operatorname{GL}(2, k)$ (see [7]), using the fact that $G$ is cyclic or of even order we get that $Q$ has no loops (see [13]). Also the number of arrows from $v$ to $w$ is known to be the same as the number of arrows from $w$ to $v$ (see $[\mathbf{7}, \mathbf{1 2}])$.

We end this section by remarking that in addition to the fact that information about the McKay quiver is gotten by considering the associated finite dimensional algebra with radical square zero, this correspondence also gives new insight into the relationship between the finite dimensional path algebras of extended Dynkin diagrams (which are known as the basic tame hereditary algebras), whose radical square is zero. It was shown in $[\mathbf{1 1}]$ that some different tame hereditary algebras were related via the skew group algebra construction with cyclic groups. It now follows that any indecomposable hereditary $k$-algebra $(\operatorname{char} k=0)$ with radical 
square zero can, up to Morita equivalence, be obtained as a direct summand of a skew group algebra of the path algebra of $\cdot \rightrightarrows \cdot($ see also $[9])$.

2. Some McKay quivers. In this section we assume $k=\mathbf{C}$. If $G$ is a finite subgroup of $\operatorname{SL}(2, \mathrm{C})$, then $G$ has no pseudoreflections, that is, no element with 1 as eigenvalue of multiplicity 1 . The McKay quivers for arbitrary finite subgroups $G$ of $\mathrm{GL}(2, \mathbf{C})$ with no pseudoreflections are of special interest since they by $[\mathbf{1}]$ give AR-quivers for the reflexive modules over the fixed ring $\mathbf{C}[[X, Y]]^{G}$. At the same time illustrating our main result, we give some classes of examples of McKay quivers containing the finite subgroups of $\mathrm{GL}(2, \mathrm{C})$ with no pseudoreflections (see $[\mathbf{3}]$ for a list of these groups). It is convenient to first introduce the following constructions of quivers.

For a tree $T$ and a positive integer $s$, the quivers $(T, s)$ and $[T, s]$ are defined as follows. In both cases the vertices are $\{(v, i) ; v \in T ; 0 \leq i<s\}$. For each edge $\dot{v}_{i}$ in $_{w} T$ we have arrows $(v, i+1) \rightarrow(w, i)$ and $(w, i+1) \rightarrow(v, i)$ in $(T, s)$, where $v$ addition is modulo $s$. Assigning + or - to the vertices of $T$ in such a way that neighbours have opposite sign, we have for each $\underset{v}{-}-\underset{w}{+}$ in $T$, arrows $(v, i) \rightarrow(w, i)$ and $(w, i+1) \rightarrow(v, i)$ in $[T, s]$. (In the terminology of $[\mathbf{1 2}],[T, s]$ is $Z \vec{T} / G$, where $\vec{T}$ is a quiver with $|\vec{T}|=T$, and $G$ is the cyclic group generated by the automorphism $\tau^{s}$.)

We have the following properties of these quivers.

LEMMA 6. (a) $(T, 2 m)$ is the disjoint union of two copies of $[T, m]$.

(b) If $T$ is connected, then $[T, m]$ is connected.

(c) If $m$ is odd, then $(T, m)$ and $[T, m]$ are isomorphic.

Proof. (a) Assign signs to the vertices of $T$ such that neighbours have different signs. Let

$$
\begin{aligned}
& Q_{1}=\{(v, i) ; v \text { positive, } i \text { even or } v \text { negative, } i \text { odd }\} \\
& Q_{2}=\{(v, i) ; v \text { negative, } i \text { even or } v \text { positive, } i \text { odd }\}
\end{aligned}
$$

Then $(T, 2 m)$ is the disjoint union of the quivers $\tilde{Q}_{1}$ and $\tilde{Q}_{2}$, where $\tilde{Q}_{i}$ is the full subquiver of $(T, 2 m)$ with vertex set $Q_{i}$. We get an isomorphism $Q_{1} \rightarrow[T, m]$ by sending $(v, 2 i)$ to $(v, i)$ if $v$ is positive and $(w, 2 i+1)$ to $(w, i)$ is $w$ is negative. Similarly we get an isomorphism $Q_{2} \rightarrow[T, m]$.

(b) If we have an edge $\underset{v}{-}-\underset{w}{+}$, then $(v, i)$ and $(v, j)$ are connected in $[T, m]$ by $(v, i) \rightarrow(w, i) \rightarrow(v, i-1) \stackrel{v}{\rightarrow}(w, i-1) \rightarrow \cdots \rightarrow(v, j)$.

(c) Let $m=2 t+1$ and define a map $\alpha:(T, m) \rightarrow[T, m]$ by

$$
\begin{aligned}
& \alpha(w, 2 i)=(w, t+i), \quad \alpha(w, 2 i-1)=(w, i) \quad \text { if } w \text { is positive, } \\
& \alpha(v, 2 i)=(v, i), \quad \alpha(v, 2 i-1)=(v, t+i-1) \quad \text { if } v \text { is negative. }
\end{aligned}
$$

The finite nontrivial subgroups of $\operatorname{GL}(2, \mathrm{C})$ having no pseudoreflections are up to conjugacy known to be of one of the following types (see $[3]$ ). Here $D$ denotes a finite noncyclic subgroup of $\operatorname{SL}(2, \mathbf{C})$ and $Z_{m}$ the finite cyclic subgroup of the center $Z$ of $\mathrm{GL}(2, \mathrm{C})$ of order $m$.

(1) $G$ is cyclic of order $n$ with generator $\left(\begin{array}{cc}\xi & 0 \\ 0 & \xi^{q}\end{array}\right)$ for a primitive $n$th root of unity $\xi$. 
(2) $D Z_{m}=\left\{d z ; d \in D, z \in Z_{m}\right\}$.

(3) A binary dihedral group $D \subset \mathrm{SL}(2, \mathrm{C})$ has a normal cyclic subgroup $A$ of index 2. Consider the group $H \subset D \times Z_{2 m}$ defined by

$$
H=\left\{(d, z) ; d \in D, z \in Z_{2 m}, d+A=z+Z_{m} \text { in } Z_{2}\right\} .
$$

We have a natural map $H \subset D \times Z_{2 m} \rightarrow \mathrm{GL}(2, \mathbf{C})$, and $\bar{H}=\operatorname{Im} H$ is a subgroup of $\mathrm{GL}(2, \mathbf{C})$.

(4) $\bar{H}=\operatorname{Im} H \subset \mathrm{GL}(2, \mathrm{C})$, where $H \subset D \times Z_{3 m}$ for a binary tetrahedral group $D$ having a normal binary dihedral subgroup $A$ of index 3 , such that $H=\{(d, z) ; d \in$ $\left.D, z \in Z_{3 m}, \phi_{1}(d+A)=\phi_{2}\left(z+Z_{m}\right)\right\}$, where $\phi_{1}: D / A \rightarrow Z_{3}$ and $\phi_{2}: Z_{3 m} / Z_{m} \rightarrow Z_{3}$ are isomorphisms.

The following computations are interesting in connection with almost split sequences, in addition to illustrating our theorem.

PROPOSITION 7. Let $T_{D}=T$ be the extended Dynkin diagram corresponding to the McKay quiver of a finite subgroup $D$ of $\mathrm{SL}(2, \mathbf{C})$.

(a) The McKay quiver for $Z_{n} \rightarrow\left\langle\left(\begin{array}{cc}\xi & 0 \\ 0 & \xi^{q}\end{array}\right)\right\rangle \subset \mathrm{SL}(2, \mathbf{C})$, where 1 is sent to the generator and $\xi$ is a primitive $n$th root of 1 , has $n$ vertices $v_{0}, \ldots, v_{n-1}$ and arrows $v_{t} \rightarrow v_{t-1}, v_{t} \rightarrow v_{t-q}$ (with addition modulo $n$ ).

(b) The McKay quiver for $D \times Z_{m} \rightarrow \mathrm{GL}(2, \mathrm{C})$ is $(T, m)$.

(c) The McKay quiver for $D Z_{2 m} \subset \mathrm{GL}(2, \mathrm{C})$ is $[T, m]$.

(d) If $H \subset D \times Z_{2 m}$ is the group defined in (3), then the McKay quiver for $H \rightarrow \mathrm{GL}(2, \mathbf{C})$ is $(T, m)$.

(e) The McKay quiver for $\bar{H}=\operatorname{Im} H \rightarrow \mathrm{GL}(2, \mathrm{C})$ is $[T, m]$, with $H \subset D \times Z_{4 m}$ as in (3).

(f) If $H \subset D \times Z_{3 m}$ is as in (4), the McKay quiver for $H \rightarrow \mathrm{GL}(2, \mathbf{C})$ is the following: The vertices are $\{(u, i) ; 0 \leq i<m\},\{(v, i) ; 0 \leq i<3 m\},\{(w, i) ; 0 \leq$ $i<3 m\}$, and we have arrows

$$
\begin{aligned}
& (u, i) \rightarrow(v, i-1), \quad(u, i) \rightarrow(v, i-1+m), \quad(u, i) \rightarrow(v, i-1+2 m), \\
& (v, i) \rightarrow(u, i-1), \quad(v, i) \rightarrow(w, i-1), \quad(w, i) \rightarrow(v, i-1)
\end{aligned}
$$

(where addition is modulo $m$ or $3 m$ ).

(g) The McKay quiver for $\bar{H}=\operatorname{Im} H \hookrightarrow \mathrm{GL}(2, \mathbf{C})$, with $H \subset D \times Z_{6 m}$ as in (4), is the following: The vertices are $\{(u, i) ; 0 \leq i<m\},\{(v, i) ; 0 \leq i<$ $3 m\},\{(w, i) ; 0 \leq i<3 m\}$, and we have arrows

$$
\begin{aligned}
& (u, i) \rightarrow(v, i-1), \quad(u, i) \rightarrow(v, i-1+m), \quad(u, i) \rightarrow(v, i-1+2 m), \\
& (v, i) \rightarrow(u, i), \quad(v, i) \rightarrow(w, i-1), \quad(w, i) \rightarrow(v, i) .
\end{aligned}
$$

ProOF. (a) With natural notation, $V_{0}=\mathbf{C}, V_{\xi}, \ldots, V_{\xi^{n-1}}$ are the irreducible representations, and $V=V_{\xi} \amalg V_{\xi^{q}}$. It is then easy to see that the McKay quiver has the desired form.

(b) Let $V^{\prime}$ be the two-dimensional representation given by $D \subset \mathrm{GL}(2, \mathrm{C})$. Let $A_{0}=\mathrm{C}, A_{\xi}, \ldots, A_{\xi^{m-1}}$ be the irreducible representations of $Z_{m}$, where $\xi$ denotes a primitive $n$th root of 1 such that 1 in $Z_{m}$ is sent to $\left(\begin{array}{ll}\xi & 0 \\ 0 & \xi\end{array}\right)$ in the inclusion $Z_{m} \rightarrow$ $\mathrm{GL}(2, \mathbf{C})$. Let $V_{0}=\mathbf{C}, V_{1}, \ldots, V_{n-1}$ denote the irreducible representations of $D$. Then the two-dimensional representation given by $D \times Z_{m} \rightarrow \mathrm{GL}(2, \mathbf{C})$ is $V=$ $V^{\prime} \otimes A_{\xi}$, and the irreducible representations of $D \times Z_{m}$ are $V_{j} \otimes A_{\xi} i ; 0 \leq j<$ 
$n, 0 \leq i<m$. If $V_{i} \mid V^{\prime} \otimes V_{j}$, then $V_{i} \otimes A_{\xi^{t}} \mid\left(V^{\prime} \otimes A_{\xi}\right) \otimes\left(V_{j} \otimes A_{\xi^{t-1}}\right)$, and this proves (b).

(c) By (b) we know that the McKay quiver for $D \times Z_{2 m} \rightarrow \mathrm{GL}(2, \mathrm{C})$ is $\left(T_{D}, 2 m\right)$, which by Lemma 6 is a disjoint union of two copies of $\left[T_{D}, m\right]$, each of which is indecomposable. The McKay quiver for $D Z_{2 m}$ is clearly a subquiver of the McKay quiver for $D \times Z_{2 m}$. We only need show that it is proper, and that if $a \rightarrow b$ is in the McKay quiver for $D \times Z_{2 m}$ and $b$ is in the subquiver, then $a$ is also. $Z \cap \mathrm{SL}(2, \mathbf{C})=\left\langle\left(\begin{array}{cc}-1 & 0 \\ 0 & -1\end{array}\right)\right\rangle \simeq Z_{2}$, and in our case $\left(\begin{array}{cc}-1 & 0 \\ 0 & -1\end{array}\right)$ is in both $D$ and $Z_{2 m}$, so that the kernel of the natural map $D \times Z_{2 m} \rightarrow \mathrm{GL}(2, \mathrm{C})$ is isomorphic to $Z_{2}$. Clearly there are some representations on which $Z_{2}$ does not act trivially. $Z_{2}$ acts trivially on the two-dimensional representation $V$, and hence if $Z_{2}$ acts trivially on some $W_{i}$, it acts trivially on every irreducible summand of $V \otimes W_{i}$. This finishes the proof of (c).

(d) Let $H \subset D \times Z_{2 m}=G$ be the subgroup of index 2 defined before. We first show that the irreducible representations of $G$ stay irreducible when restricted to $H$. This is clearly the case for the one-dimensional ones. Now it is well known that the others are two-dimensional (see [7]). Let $G \rightarrow \mathrm{GL}(2, \mathbf{C})$ be such a representation. Since it is two-dimensional, the commutator subgroup $[G, G]$ of $G$ is not contained in the kernel. To show that the representation given by the restriction map $H \rightarrow \mathrm{GL}(2, \mathbf{C})$ does not decompose into the direct sum of two one-dimensional representations, it is sufficient to show that $[G, G] \subset[H, H]$. To see this, let $(d, z)$ and $\left(d^{\prime}, z^{\prime}\right)$ be in $G$. Then $(d, z)\left(d^{\prime}, z^{\prime}\right)\left(d^{-1}, z^{-1}\right)\left(d^{\prime-1}, z^{\prime-1}\right)=\left(d d^{\prime} d^{-1} d^{\prime-1}, 1\right)$. By the definition of $H$ we can choose $z_{1}$ and $z_{1}^{\prime}$ in $Z_{2 m}$ such that $\left(d, z_{1}\right)$ and $\left(d^{\prime}, z_{1}^{\prime}\right)$ are in $H$. Then $\left(d, z_{1}\right)\left(d^{\prime}, z_{1}^{\prime}\right)\left(d^{-1}, z_{1}^{-1}\right)\left(d^{\prime-1}, z_{1}^{\prime-1}\right)=\left(d d^{\prime}, d^{-1} d^{\prime-1}, 1\right)$, which proves the claim.

Denote by $\alpha$ the representation of $Z_{2 m}$ given by $Z_{2 m} \rightarrow Z_{2 m} / Z_{m} \simeq Z_{2} \hookrightarrow$ $\mathrm{SL}(2, \mathbf{C})$, and by $\beta$ the representation of $D$ given by $D \rightarrow D / A \simeq Z_{2} \hookrightarrow \operatorname{SL}(2, \mathbf{C})$. If $A_{i}$ is an irreducible representation of $Z_{2 m}$ and $V_{j}$ an irreducible representation of $D$, then $\left(A_{i} \otimes \alpha\right) \otimes\left(V_{j} \otimes \beta\right)$ is not isomorphic to $A_{i} \otimes V_{j}$, but is clearly isomorphic to $A_{i} \otimes$ $V_{j}$ when restricted to $H$. That no other nonisomorphic irreducible representations of $G$ become isomorphic when restricted to $H$ follows from the fact that the number of conjugacy classes of $H$ is equal to half the number of conjugacy classes of $G$. To see this, we first observe that since $H$ is normal in $G$, each $H$-conjugacy class in $H$ is a $G$-conjugacy class in $G$. Let $(d, z)$ be an element of $G$ not in $H$. Multiplication by $\left(1, z^{\prime}\right)$, where $z^{\prime}$ is a generator for $Z_{2 m}$, gives a one-one correspondence between conjugacy classes outside and inside $H$. The McKay quiver for $D \times Z_{2 m}$ is $\left(T_{D}, 2 m\right)$, where $T_{D}$ is of the form

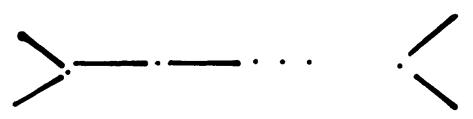

The end vertices of $T_{D}$ correspond to the one-dimensional irreducible representations of $D$, and $-\otimes \beta$ induces an automorphism of $T_{D}$ which clearly moves the end vertices. By considering, for example, the number of irreducible representations of $A$, it is easy to see that $-\otimes \beta$ does not move the irreducible two-dimensional representations. These comments determine the automorphism of $T_{D}$, and it is now not hard to see that the McKay quiver for $H$ is $\left(T_{D}, m\right)$.

(e) We now have $H \subset Z_{4 m} \times D$. The element $\left(\left(\begin{array}{cc}-1 & 0 \\ 0 & -1\end{array}\right)\right),\left(\left(\begin{array}{cc}-1 & 0 \\ 0 & -1\end{array}\right)\right)$ is contained in $H$, so that the kernel of $H \rightarrow \mathrm{GL}(2, \mathbf{C})$ is isomorphic to $Z_{2}$. As in (b) it follows 
from the fact that the McKay quiver for $H$ is $\left(T_{D}, 2 m\right)$ that the McKay quiver for $\bar{H}$ is $\left[T_{D}, m\right]$.

(f) The proof is similar to the proof of (d). To show that an irreducible representation of $D \times Z_{3 m}$ stays irreducible, we use that the irreducible representations of $D$, and hence of $D \times Z_{3 m}$, have dimension at most 3 (see [7]), and we use Clifford theory to conclude that if the restriction of a 3-dimensional representation does not decompose into a direct sum of one-dimensional representations, then it is irreducible. For in particular the different summands must have the same dimension (see [5]). We now consider the representation $\alpha$ of $Z_{3 m}$ given by $Z_{3 m} \rightarrow Z_{3 m} / Z_{m} \simeq Z_{3} \rightarrow \mathrm{GL}(2, \mathbf{C})$, where 1 in $Z_{3}$ is sent to $\left(\begin{array}{ll}\xi & 0 \\ 0 & \xi\end{array}\right)$ for $\xi$ a primitive third root of 1 . And we consider the representation $\beta$ of $D$ given similarly with the same $\xi$, and use that $\alpha^{2} \otimes \beta$ and $\alpha \otimes \beta^{2}$ have trivial restriction to $H$. $-\otimes \beta$ gives an automorphism of the McKay quiver

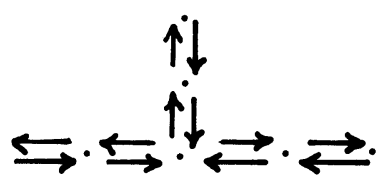

of $D$, which clearly moves all one-dimensional representations. Since these are known to correspond to the three end vertices (see [7]), the automorphism is determined (up to $\beta^{2}$ ). Considering the identifications indicated, it is easy to see that $D \times Z_{3 m}$ has the claimed McKay quiver.

(g) This is similar to (e), since it is easy to see that the McKay quiver for $D \times Z_{6 m}$ is the disjoint union of two isomorphic components. (In the terminology of [12] we have $Z \overrightarrow{\tilde{E}}_{6} / G$, where $\left|\overrightarrow{\tilde{E}}_{6}\right|=\tilde{E}_{6}$ and $G$ is the cyclic group determined by $\tau^{m}$ and an automorphism of order 3 of $\tilde{E}_{6}$.)

For the cyclic subgroups of $\mathrm{GL}(2, \mathrm{C})$ we easily see that the underlying graph of the separated McKay quiver is the union of diagrams of the form $\tilde{A}_{t}$. Further, the underlying graph of the separated quiver of $(T, m)$ and $[T, m]$ is $2 m$ copies of $T$. In cases (f), (g) we get $2 m$ copies of $\tilde{E}_{6}$.

We point out that the AR-quiver has a translation $\tau$ giving the correspondence between the end terms of almost split sequences. The McKay quiver also has a natural translation $\tau$ given by $\tau\left(V_{i}\right)=\operatorname{det} \otimes V_{i}$, where det denotes the associated determinantal representation. Hence $\tau$ is the identity for a subgroup of $\operatorname{SL}(2, \mathbf{C})$. Considering $\tau$ defined on all representations except the trivial one, it follows from [1] that the McKay quiver and the corresponding AR-quiver are isomorphic as translation quivers for subgroups of $\mathrm{GL}(2, \mathbf{C})$ having no pseudoreflections. In the above cases it is not hard to compute $\tau$, but we do not include this computation here.

We end this section with two other examples pointed out to us by M. Isaacs. Here the McKay quivers are not isomorphic to AR-quivers for reflexive modules over any $\mathbf{C}[[X, Y]]^{G}$. Other examples are found in $[\mathbf{6}]$.

There is an embedding $\operatorname{GL}(2,3) \subset \mathrm{GL}(2, \mathbf{C})$, with associated McKay quiver: 


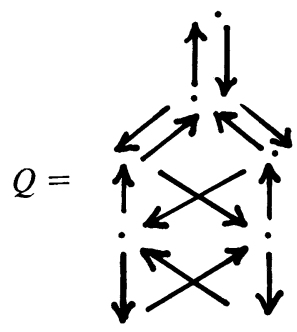

Here $|\bar{Q}|=\tilde{E}_{7} \cup \tilde{E}_{7}$.

There is a map $S_{4} \rightarrow \mathrm{GL}(2, \mathrm{C})$ with associated McKay quiver:
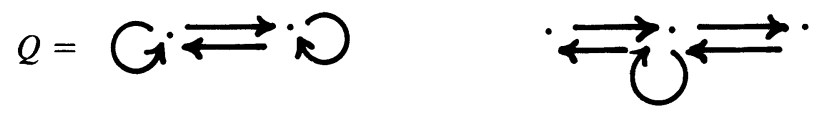

Here $|\bar{Q}|=\tilde{A}_{3} \cup \tilde{D}_{5}$.

We remark that in this section we can replace $\mathbf{C}$ by an algebraically closed field $k$, and consider only the subgroups of $\operatorname{GL}(2, k)$ whose order is not divisible by the characteristic of $k$.

\section{REFERENCES}

1. M. Auslander, Rational singularities and almost split sequences, Trans. Amer. Math. Soc. (to appear).

2. M. Auslander, M. Platzeck and I. Reiten, Periodic modules over weakly symmetric algebras, J. Pure Appl. Algebra 11 (1977), 279-291.

3. E. Brieskorn, Rationale Singularitäten komplexer Flächen, Invent. Math. 4 (1968), 336-358.

4. V. Dlab and C. Ringel, Indecomposable representations of graphs and algebras, Mem. Amer. Math. Soc. No. 173 (1976).

5. L. Dornhoff, Group representation theory, Dekker, New York, 1971.

6. G. Gonzalez-Sprinberg and J. Verdier, Construction géométrique de la correspondance de McKay, Ann. Sci. École Norm. Sup. (4) 16 (1983), 409-449.

7. D. Happel, U. Preisel and C. Ringel, Binary polyhedral groups and Euclidean diagrams, Manuscripta Math. 31 (1980), 317-329.

8. H. Knörrer, Untitled preprint.

9. H. Lenzing, Polyhedral groups and the geometric study of tame hereditary algebras (preprint).

10. J. McKay, Graphs, singularities and finite groups, Proc. Sympos. Pure Math., vol. 37, Amer. Math. Soc., Providence, R. I., 1980, pp. 183-186.

11. I. Reiten and C. Riedtmann, Skew group algebras in the representation theory of artin algebras, J. Algebra 92 (1985).

12. C. Riedtmann, Algebren, Darstellungsköcher, Überlagerungen und zurück, Comment. Math. Helv. 55 (1980), 199-224.

13. R. Steinberg, Subgroups of $S U_{2}$ and Dymkin diagrams, Pacific J. Math. 118 (1985), 587-598.

Department of Mathematics, Brandeis University, Waltham, MassachuSETTS 02254

INSTITUTT FOR MATEMATIKK OG STATISTIKK, UNIVERSITETET I TRONDHEIM, AVH, 7055 DRAGVOLL, NORWAY 